\title{
Previsões de Precipitação de Modelos Atmosféricos como Subsídio à Operação de Sistemas de Reservatórios
}

\author{
Pedro Ludovico Bozzini ${ }^{1}$ (D), Arisvaldo Vieira Méllo Júnior ${ }^{1}$ \\ ${ }^{1}$ Laboratório de Sistemas de Suporte a Decisões, Departamento de Engenharia Hidráulica e \\ Ambiental, Escola Politécnica, Universidade de São Paulo, São Paulo, SP, Brasil.
}

Recebido em: 12 de Junho de 2019 - Aceito em: 17 de Fevereiro de 2020

\begin{abstract}
Resumo
Modelos atmosféricos globais e regionais são importantes ferramentas para a gestão de recursos hídricos. Com a intenção de realizar previsão de vazões e subsidiar a operação de sistemas de abastecimento, esse estudo avaliou as previsões de precipitação de três modelos atmosféricos. Foram analisadas as previsões dos modelos ETA (resolução horizontal de $40 \mathrm{~km}$, horizonte de 10 dias), BAM (resolução horizontal de $20 \mathrm{~km}$, horizonte de 10 dias) e WRF (resolução horizontal de $5 \mathrm{~km}$, horizonte de 3 dias) para as bacias do Sistema Cantareira, no Sudeste brasileiro. As previsões foram comparadas com observações de pluviômetros e de radar. Foram avaliados o coeficiente de correlação de Pearson, eficiência modificada de Kling-Gupta, coeficiente angular da regressão linear entre previsões e observações, REQM e PBIAS, além dos índices categóricos fBIAS, POD e FAR. Verificou-se que as correlações tendem a ser mais fortes nos primeiros dias de previsão (até o segundo dia à frente). As maiores correlações foram encontradas comparando valores acumulados para todo o horizonte de previsão. O modelo ETA apresentou forte tendência a subestimar as observações e o modelo BAM, de superestimá-las. O modelo WRF apresentou uma tendência leve de subestimação. A previsão de precipitação com modelos globais e regionais é um importante subsídio à operação de sistemas de reservatórios, entretanto, é essencial conhecer o comportamento dessas previsões a fim de minimizar imprecisões e maximizar seu valor na tomada de decisão.
\end{abstract}

Palavras-chave: previsão de precipitação, modelo ETA, modelo WRF, modelo BAM, Sistema Cantareira.

\section{Atmospheric Model Precipitation Forecast Analysis to Support Reservoir Systems Operation}

\begin{abstract}
Global and regional atmospheric models are important tools for water resources management. Aiming to provide river flow forecast and to support water supply systems operation, this study evaluated the precipitation forecasts from three atmospheric models. Forecasts from ETA (horizontal resolution of $40 \mathrm{~km}, 10$ days range), BAM (horizontal resolution of $20 \mathrm{~km}, 10$ days range) and WRF (horizontal resolution of $5 \mathrm{~km}, 3$ days range) models were analyzed for the Cantareira system basins, on Brazil's southwestern region. The forecasts were compared to rain gauge and radar observations. Different performance indices were used: Pearson's correlation coefficient, modified Kling-Gupta efficiency, slope for the linear regression between forecasts and observations, RMSE and PBIAS, in addition to categorical indices fBIAS, POD e FAR. It was found that the correlations were stronger for the first days of the forecast. The strongest correlations were found when comparing the accumulated precipitations for the whole forecast period. Bias analysis indicated ETA strongly underestimated observations, while BAM strongly overestimated, and WRF slightly underestimated. Global and regional atmospheric models rainfall forecasts are an important input for reservoir operation. It's essential, though, to understand its behavior to minimize inaccuracies and maximize its value in decision making.
\end{abstract}

Keywords: precipitation forecast, ETA model, WRF model, BAM model, Cantareira system.

Autor de correspondência: Pedro Ludovico Bozzini, pedro.bozzini@alumni.usp.br. 


\section{Introdução}

O aumento da população e, por consequência, da necessidade de produção de alimentos, energia e bens de consumo provocam um aumento da demanda e dos conflitos pelo uso da água (Schardong, 2009). Soma-se a isso a crescente importância da questão ambiental, que traz à discussão os impactos causados pela expansão dos sistemas de aproveitamento dos recursos hídricos. Dessa forma, tornam-se cada vez mais importantes as medidas de gestão que permitem um melhor aproveitamento das estruturas existentes.

Modelos estocásticos são bastante complexos para uso em tempo real na operação e a qualidade das previsões é alvo de críticas (Barros, 2009). Os modelos climáticos globais e regionais são, atualmente, importantes ferramentas no estudo de sistemas de recursos hídricos (Schardong, 2014) e apresentam-se como uma possível alternativa para a melhoria da operação, permitindo a tomada de decisão com base em informações para um maior horizonte de tempo.

Esse estudo está inserido no contexto de um projeto que busca desenvolver sistemas que auxiliem na operação do Sistema Cantareira (SC), operado pela Sabesp. O SC é o maior sistema de abastecimento da Região Metropolitana de São Paulo, com capacidade de $33 \mathrm{~m}^{3} / \mathrm{s}$ (SABESP, 2009). A outorga vigente foi definida pela resolução conjunta ANA/DAEE no 926 (ANA/DAEE, 2017b) e regulamentada pela resolução conjunta ANA/DAEE $\mathrm{n}^{\circ} 925$ (ANA/DAEE, 2017a). Os documentos estabelecem regras operacionais, como a exigência de vazões mínimas em três pontos a jusante das represas. É importante para a operação do sistema o conhecimento das contribuições naturais das bacias a jusante, pois determinam a vazão que deverá ser descarregada em cada barragem para atendimento da norma. A qualidade da previsão de curto prazo da precipitação é útil para a estimativa das vazões afluentes aos reservatórios por meio de modelagem hidrológica. Nesse sentido, Murphy (1993) discute o significado de uma "boa previsão", sugerindo que uma forma de uma previsão ser boa é possibilitando a tomada de melhores decisões.

O objetivo desse trabalho é avaliar, para as bacias relacionadas ao $\mathrm{SC}$, o desempenho das previsões de precipitação dos modelos ETA, WRF e BAM. Os resultados obtidos servirão de subsídio para o desenvolvimento de previsões de vazão e auxílio na operação do SC.

\section{Materiais e Métodos}

\subsection{Região de estudo}

$\mathrm{O}$ estudo foi desenvolvido na região que engloba as represas do sistema Cantareira e a região a jusante, onde estão localizados os pontos de controle definidos pela outorga. As bacias foram delimitadas a partir da Base Hidrográfica Ottocodificada Multiescalas 2013, disponibilizada pela ANA. As bacias foram agregadas de modo que cada estrutura de interesse estivesse associada a uma única bacia. Cada bacia dispunha de ao menos um pluviômetro, cujos dados foram utilizados nesse estudo A Fig. 1 ilustra as bacias e postos, a Tabela 1 traz informações sobre as bacias.

\subsection{Modelo ETA}

O Modelo ETA teve sua operação iniciada no National Centers for Environmental Prediction (NCEP) (Mesinger et al., 1988; Black, 1994). No Brasil, o modelo é mantido pelo Centro de Previsão do Tempo e Estudos Climáti$\cos$ (CPTEC) do Instituto Nacional de Pesquisas Espaciais (INPE) que o utiliza para complementar a previsão numérica desde 1996 (Chou, 1996), tendo passado por diversas melhorias desde então (Messinger et al., 2012).

Segundo Chou (1996), sendo regional, o ETA se propõe a prever mais detalhadamente fenômenos ligados a sistemas de mesoscala, como frentes, tempestades severas e decorrências da orografia.

Moura (2010) verificou que o modelo tende a superestimar as precipitações nas primeiras 24 horas, sendo esse desvio maior principalmente na região Norte do Brasil. Cataldi (2007) constatou que o modelo tende a subestimar precipitações mais intensas (superior a $10 \mathrm{~mm}$ ). Esses estudos evidenciam que a tendência do modelo pode variar em função do horizonte de previsão, da região analisada e do volume de chuva observado.

As previsões foram obtidas para resolução espacial de $40 \mathrm{~km}$ e horizonte de 10 dias. No momento da escrita deste artigo, as previsões para essa resolução não estavam mais à disposição do público. Foram analisadas previsões de 09/01/2018 a 29/10/2018 para as bacias do Jaguari/ Jacareí, Atibainha, Cachoeira e Paiva Castro. Para as demais bacias, incluídas posteriormente no estudo, foram analisadas previsões de 08/03/2018 a 29/10/2018.

\subsection{Modelo WRF}

O Weather Research and Forecasting Model (modelo WRF) foi desenvolvido por agências e universidades americanas para dar suporte à pesquisa e aplicações operacionais. O modelo, em sua terceira versão, é descrito em detalhes por Skamarock (2018). No Brasil, teve sua operação iniciada na ocasião das Olimpíadas de 2016, para prever ondas e correntes (INPE, 2016). Desde então, tem sido aplicado em diversos estudos (Cardoso, 2018; Pellegrini, 2019; Loriato, 2018).

As previsões foram obtidas para resolução espacial de $5 \mathrm{~km}$ e horizonte de 3 dias. Foram analisadas previsões de 22/01/2019 a 21/05/2019 para todas as bacias.

\subsection{Modelo BAM}

O Modelo Atmosférico Brasileiro (BAM - Brazilian Atmospheric Model) é um modelo global operado pelo CPTEC/INPE desde 2016. Seus resultados são utili- 


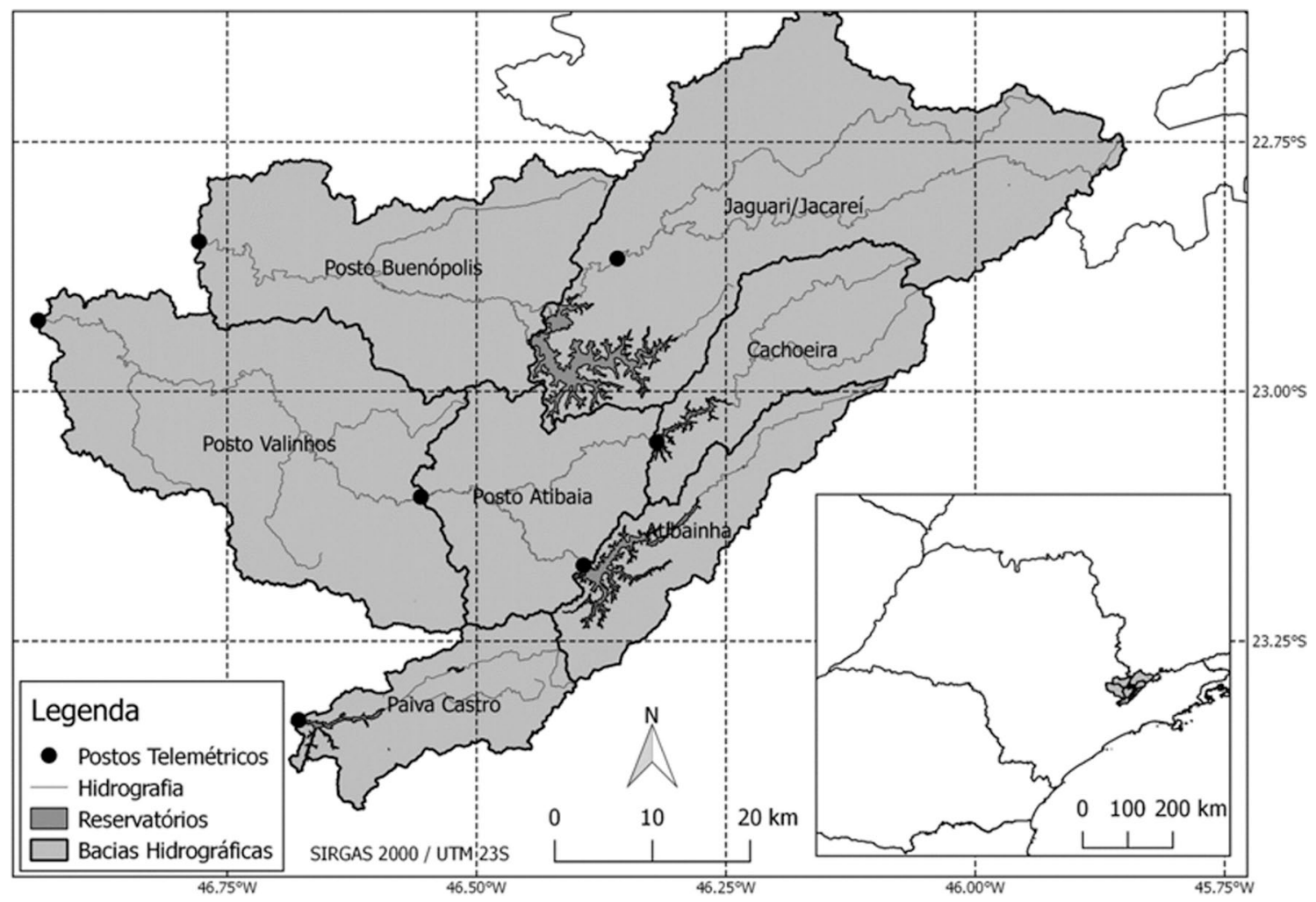

Figura 1 - Bacias hidrográficas e pluviômetros considerados no estudo.

Tabela 1 - Bacias hidrográficas utilizadas no estudo e suas respectivas áreas de drenagem.

\begin{tabular}{lcccccc}
\hline Jaguari/Jacareí & Cachoeira & Atibainha & Paiva Castro & Posto Buenópolis & Posto Valinhos & Posto Atibaia \\
\hline $1239,34 \mathrm{~km}^{2}$ & $392,44 \mathrm{~km}^{2}$ & $315,16 \mathrm{~km}^{2}$ & $300,61 \mathrm{~km}^{2}$ & $715,53 \mathrm{~km}^{2}$ & $436,33 \mathrm{~km}^{2}$ & $982,71 \mathrm{~km}^{2}$ \\
\hline
\end{tabular}

zados como condição inicial de modelos regionais (INPE, 2016). É descrito em detalhes por Figueroa (2016), que apresenta o BAM como solução para deficiências de modelos utilizados anteriormente. Dentre as vantagens, Figueroa destaca a melhora na previsão para o sudeste do Brasil em decorrência de uma maior resolução horizontal, modelando melhor eventos relacionados com orografia complexa.

As previsões foram obtidas para resolução espacial de $20 \mathrm{~km}$ e horizonte de 10 dias. Foram analisadas previsões de 01/01/2019 a 22/05/2019 para todas as bacias.

\subsection{Precipitação estimada com radar meteorológico}

Postos pluviométricos são instrumentos adequados para medir a intensidade da chuva, mas não possuem boa representatividade espacial (Pereira Filho, 1995). Buscouse, então, observações de precipitação que levassem em conta a distribuição espacial na bacia.

O radar meteorológico de São Paulo é operado pelo Sistema de Alerta a Inundações de São Paulo (SAISP) e fica localizado em Biritiba Mirim - SP, na barragem de Ponte Nova. As estimativas de precipitação são obtidas a partir dos campos de refletividade no formato "Constant
Altitude Plain Position" (CAPPI) de $3 \mathrm{~km}$ de altura, com resolução espacial de $1 \times 1 \mathrm{~km}$. A transformação em taxa de precipitação é feita de acordo com a relação Z-R descrita em (Rocha Filho, 2010 e SAISP, 2019). As observações foram obtidas com resolução temporal de $10 \mathrm{~min}$. Para cada bacia, foi realizada uma média ponderada dos valores em cada quadrícula, agregados diariamente.

\subsection{Rede de pluviômetros}

Foram utilizadas estações pluviométricas operadas pelo SAISP, pertencentes à rede de monitoramento da Companhia de Saneamento Básico do Estado de São Paulo (SABESP), cujos equipamentos são pluviômetros automáticos de báscula com resolução amostral de $0,2 \mathrm{~mm}$ e resolução temporal de 10 minutos. Para cada bacia, foi utilizada uma estação pluviométrica. Os dados foram agregados para se obter as precipitações acumuladas diárias.

\subsection{Comparação entre modelos de previsão e métodos de medição da precipitação}

Para cada um dos modelos de previsão (ETA, WRF e BAM) foram realizadas comparações com as observações 
(Radar e Pluviômetro). Para cada par "previsão x observação", foram realizadas as seguintes comparações dos valores previstos com os respectivos valores observados:

- Série completa de valores previstos, independente do número de dias à frente;

- Valores previstos agrupados por número de dias à frente de quando a previsão foi realizada, isto é, todos os valores previstos para o dia seguinte à rodada do modelo, depois todos os valores previstos para dois dias à frente e assim sucessivamente;

- Valores acumulados para todo o período de previsão.

Para cada comparação foram calculados diversos índices de avaliação. O coeficiente de correlação de Pearson é uma medida da relação linear entre duas variáveis aleatórias (por exemplo, série de precipitações observadas e previstas), e é calculado conforme a Eq. (1):

$$
r=\frac{\sum(x-\bar{x})(y-\bar{y})}{\sqrt{\sum(x-\bar{x})^{2} \sum(y-\bar{y})^{2}}}
$$

Kessler (1994) indica o coeficiente para analisar a relação entre duas formas de se estimar uma mesma grandeza, como é o caso da precipitação medida por um pluviômetro ou por um radar. Habib (2001) alerta para que o valor seja interpretado com cautela, pois o coeficiente é sensível a valores extremos e parte da premissa de que as variáveis são normalmente distribuídas. Para grandes amostras temos, pelo teorema do limite central, que a distribuição se aproxima da normal, justificando o uso de ferramentas paramétricas (Elliot, 2007). Dessa forma, é possível avaliar a significância do coeficiente através de um teste t. A interpretação pode ser feita através de faixas (Taylor, 1990 e Mukaka, 2012), indicando correlação moderada para valores de $\mathrm{r}$ acima de 0,5 , forte para valores acima de $0,7 \mathrm{e}$ muito forte para valores acima de 0,9. Apesar dessas faixas-guia, a interpretação do valor de $r$ é insuficiente sem conhecer a natureza dos dados, tamanho da amostra e outros fatores que ajudem a interpretar a relação das variáveis (Taylor, 1990).

Nesse caso, a relação entre as variáveis é conhecida. Sendo diferentes estimativas de uma mesma grandeza, as variáveis, plotadas uma em função da outra, deveriam se alinhar em uma reta de $45^{\circ}(\mathrm{y}=\mathrm{x})$ num cenário perfeito (Kessler 1994). Nesse sentido, foi avaliada a dispersão dos valores previstos em relação aos observados, ajustando-se uma reta com intersecção na origem $(y=a * x)$. Sendo y $o$ valor previsto e $\mathrm{x}$ o observado, temos que para a $>1$, existe tendência de a previsão superestimar o valor observado. Para $a<1$, existe tendência de subestimar o valor observado.

$\mathrm{O}$ índice de eficiência modificado de Kling-Gupta (Eq. (2)), proposto por Gupta (2009) e alterado por Kling (2012), considera três diferentes componentes: a correlação linear (r), através do coeficiente de correlação de Pearson, o viés $(\beta)$, através da razão entre as médias $(\mu)$
(Eq. (3)), e a variabilidade ( $\gamma$ ), através da razão entre os coeficientes de variação, que representam a razão entre o desvio-padrão $(\sigma)$ e a média $(\sigma / \mu)$ (Eq. (4)). O índice, bem como suas componentes, possuem valor ótimo igual a 1.

$$
\begin{gathered}
K G E^{\prime}=1-\sqrt{(r-1)^{2}+(\beta-1)^{2}+(\gamma-1)^{2}} \\
\beta=\frac{\mu_{\text {prev }}}{\mu_{\text {obs }}} \\
\gamma=\frac{\sigma_{\text {prev }} / \mu_{\text {prev }}}{\sigma_{\text {obs }} / \mu_{\text {obs }}}
\end{gathered}
$$

Outras formas tradicionais de avaliação de modelos incluem a Raiz do Erro Quadrático Médio (REQM), dado pela Eq. (5), e a porcentagem de viés (PBIAS), dado pela Eq. (6). O REQM avalia a diferença média entre os valores previstos e observados, enquanto que o PBIAS indica a existência e magnitude de viés de uma amostra em relação à outra.

$$
\begin{gathered}
R E Q M=\sqrt{\frac{\sum\left(x_{\text {prev }}-y_{\text {obs }}\right)^{2}}{N}} \\
P B I A S=\frac{\sum\left(x_{\text {prev }}-y_{\text {obs }}\right)}{\sum\left(y_{\text {obs }}\right)}
\end{gathered}
$$

Esses índices levam em consideração a magnitude das diferenças. Os índices categóricos (Salio, 2015 e BaezVillanueva, 2018), por outo lado, avaliam de forma binária a ocorrência ou não de precipitação, classificando-os em categorias, conforme a Tabela 2: evento observado e previsto pelo modelo $(\mathrm{H})$, evento observado, mas não previsto (M), evento previsto pelo modelo, mas não observado (F). A ocorrência ou não de um evento é dada pelo valor de precipitação acima de um limiar estabelecido. Variando o valor desse limiar, é possível verificar o comportamento das previsões em diferentes faixas. Foram utilizados limiares variando de $0,5 \mathrm{~mm}$ a $50 \mathrm{~mm}$. Assim, definem-se os seguintes índices: frequência de viés (fBIAS, Eq. (7)), probabilidade de detecção (POD, Eq. (8)), e falso alarme (FAR, Eq. (9)). A análise conjunta desses índices é importante pois um modelo pode, por exemplo, obter um bom

Tabela 2 - Classificação de eventos para cálculo dos índices categóricos.

\begin{tabular}{lccr}
\hline & & \multicolumn{2}{c}{ Previsto } \\
\cline { 3 - 4 } & & Sim & Não \\
\hline Observado & Sim & H & M \\
& Não & F & \\
\hline
\end{tabular}


desempenho no POD por prever grandes volumes de precipitação com frequência, o que pode ser denunciado ao se avaliar também o FAR.

$$
\begin{gathered}
f B I A S=\frac{F+H}{M+H} \\
P O D=\frac{H}{M+H} \\
F A R=\frac{F}{F+H}
\end{gathered}
$$

\section{Resultados e Discussão}

A Tabela 3 mostra os valores para o coeficiente de correlação de Pearson calculado para as análises com séries completas de previsão.

O coeficiente de correlação entre a série completa de previsões levantadas e as observações é bastante baixa em todos os casos. A correlação foi mais forte ao se comparar com as observações de radar, o que é esperado, dado que tanto o radar quanto os modelos de previsão levam em consideração a espacialização da chuva. Para os casos onde o valor do coeficiente de correlação foi maior ou igual a 0,2 o teste t, com significância $99 \%$, indicou que são estatisticamente diferentes de 0 . O modelo ETA apresentou maiores valores de correlação, seguido do modelo BAM e por fim o modelo WRF. Essa comparação pode ser vista nos gráficos comparando as 4930precipitações do radar com as do modelo ETA (Fig. 2a), WRF (Fig. 2b) e BAM (Fig. 2c).

Comparando os valores agregados pelo número de dias à frente em relação à rodada do modelo, podemos verificar maior correlação nos primeiros dias de previsão, com forte decrescimento nos últimos dias, principalmente nos modelos com maior horizonte de previsão (ETA e BAM). Esse decréscimo ao longo do horizonte era esperado, conforme as análises da bibliografia consultada (Figueroa, 2016 e Moura, 2010). O modelo WRF apresentou melhora na correlação no segundo dia de previsão em 14 das 15 análises. Esse resultado está de acordo com Cataldi (2007), que explica o fato pela inicialização dos modelos, que assume valores iniciais nulos para algumas variáveis, produzindo erros nas primeiras horas de simulação, até que as variáveis atinjam valores mais próximos das observações. A Fig. 3 mostra a variação do coeficiente de correlação ao longo do horizonte de previsão para a bacia do Jaguari/Jacareí e observações do radar.
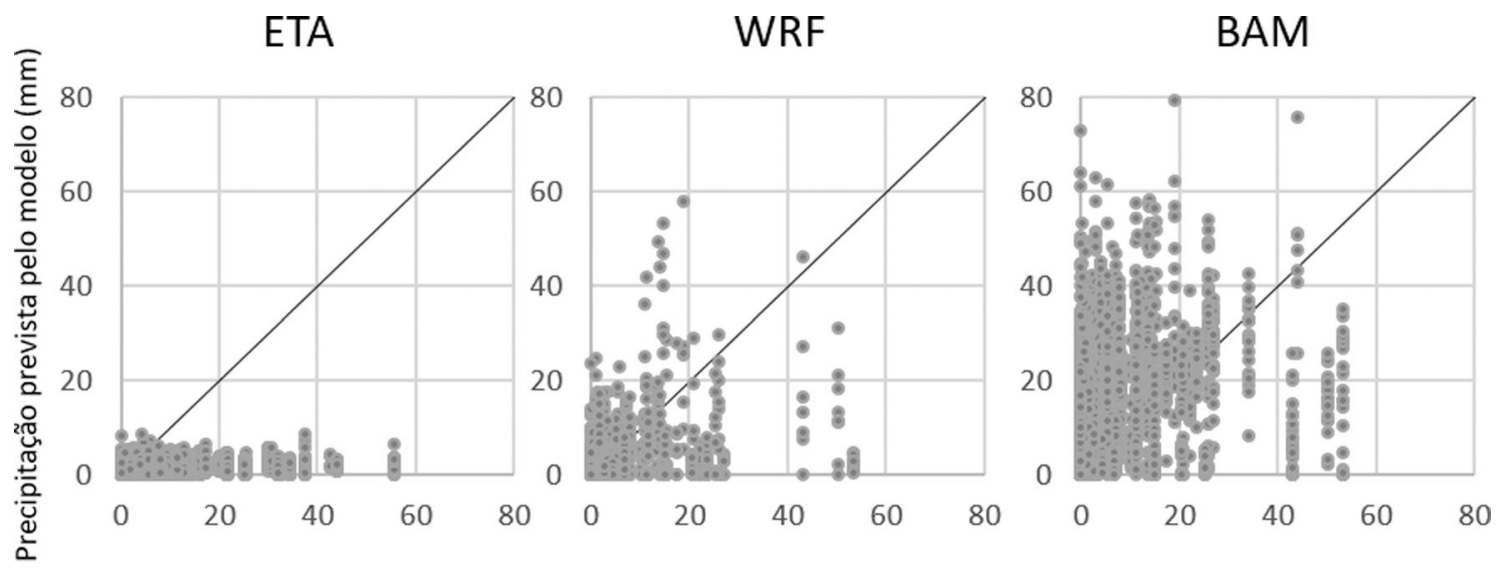

Precipitação observada pelo radar $(\mathrm{mm})$

\begin{tabular}{|c|c|c|c|c|c|c|}
\hline & \multicolumn{2}{|c|}{ ETA } & \multicolumn{2}{|c|}{ WRF } & \multicolumn{2}{|c|}{ BAM } \\
\hline & Radar & Pluviômetro & Radar & Pluviômetro & Radar & Pluviômetro \\
\hline Jaguari/Jacareí & 0,3454 & 0,2287 & 0,0231 & 0,0801 & 0,2371 & 0,1888 \\
\hline Cachoeira & 0,3293 & 0,2834 & 0,0315 & $-0,0029$ & 0,2339 & 0,2312 \\
\hline Atibainha & 0,2996 & 0,2750 & $-0,0325$ & $-0,0120$ & 0,3114 & 0,2780 \\
\hline Paiva Castro & 0,2418 & 0,2149 & 0,0053 & 0,0252 & 0,2707 & 0,2482 \\
\hline Posto Buenópolis & 0,2728 & 0,2821 & 0,1448 & 0,1142 & 0,2177 & 0,1145 \\
\hline Posto Valinhos & 0,2629 & 0,1840 & 0,0809 & 0,0510 & 0,2513 & 0,2242 \\
\hline Posto Atibaia & 0,2612 & 0,2319 & 0,0973 & 0,1113 & 0,3077 & 0,2228 \\
\hline
\end{tabular}

Figura 2 - Relação entre as precipitações observadas pelo radar e previstas pelos modelos ETA (a), WRF (b) e BAM (c).

Tabela 3 - Correlação de Pearson para as análises com série completa de previsões. 
Os maiores valores para o coeficiente de correlação foram os encontrados ao se comparar os valores acumulados para todo o horizonte de previsão com os valores acumulados observados no mesmo período. Esse resultado está de acordo com Cataldi (2007). A Fig. 6a apresenta os coeficientes de correlação para a comparação com a precipitação acumulada em todos os cenários.

As análises indicaram correlação acima de $0,7 \mathrm{em} 3$, acima de 0,6 em 19 e acima de 0,5 em 29 do total de 42 casos (3 modelos comparados com 2 fontes de dados observados em cada uma das 7 bacias). O modelo ETA, que apresentou as maiores correlações para as bacias Jaguari/Jacareí e Cachoeira, apresentou correlação bastante fraca para as bacias Posto Valinhos (Radar e Pluviômetro) e Posto Atibaia (Radar). O modelo WRF apresentou maior consistência entre os casos analisados, com coeficientes de correlação acima de 0,5 em todos os casos e acima de 0,6 em 11 dos 14 casos. O modelo BAM também apresentou uma amplitude menor de variação do coeficiente de correlação, mas com valores inferiores aos encontrados para o modelo WRF.
Os gráficos a seguir ilustram as relações verificadas para as precipitações acumuladas na bacia Jaguari/Jacareí para o modelo ETA (Fig. 4a), WRF (Fig. 4b) e BAM (Fig. 4c). Neles é possível ver de forma mais clara como o aumento no coeficiente de correlação para as precipitações acumuladas reflete num maior alinhamento dos valores.

Os gráficos também permitem avaliar visualmente $\mathrm{o}$ impacto do coeficiente angular da regressão linear. A Tabela 4 mostra o coeficiente angular para todos os cenários de comparação da precipitação acumulada. Os resultados mostram uma forte tendência do modelo ETA de subestimar os valores de precipitação e uma forte tendência do modelo BAM de superestimar esses valores. $\mathrm{O}$ modelo WRF indicou leve tendência de subestimar os valores. A análise, no entanto, requer cautela. Como o gráfico (Fig. 2b) ilustra, em uma parcela significativa dos pontos o modelo WRF indicou um valor maior que o observado. Esse caso é um exemplo da importância da análise conjunta da correlação e do viés. Uma forte correlação não dá informações sobre a proporção da relação entre as duas variáveis, enquanto uma fraca correlação

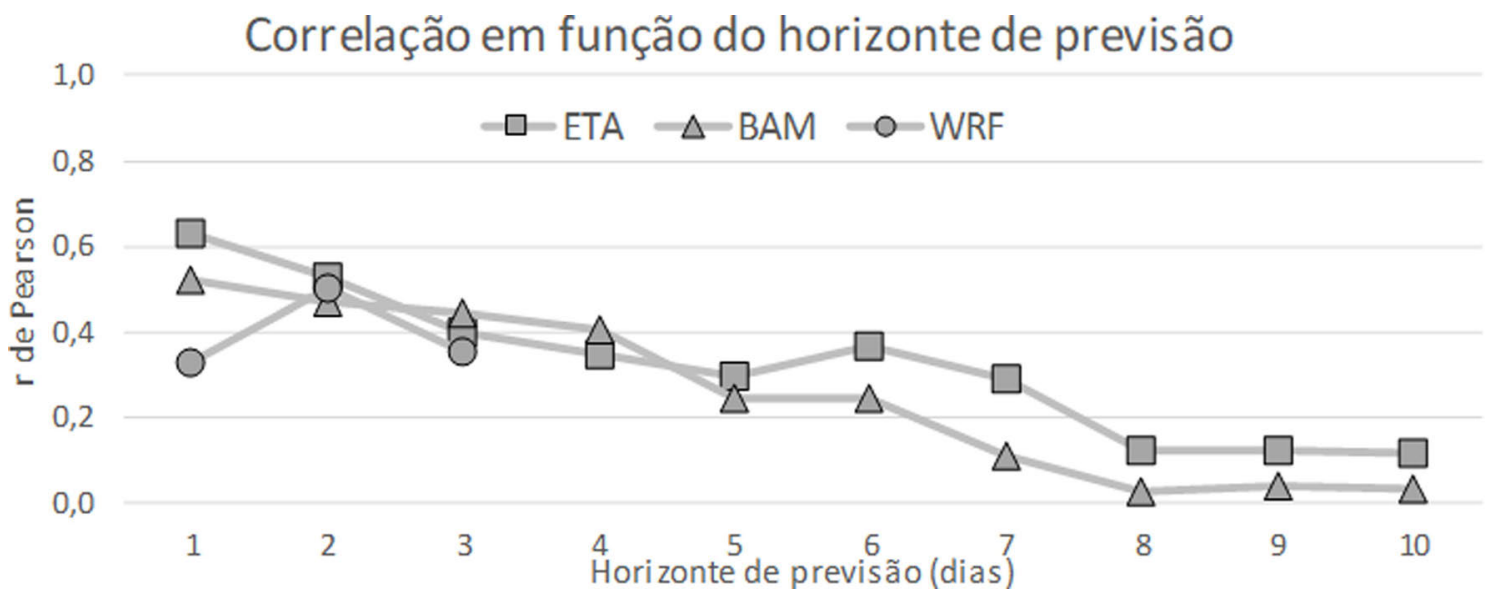

Figura 3 - Correlação com as observações de radar em função do horizonte de previsão.
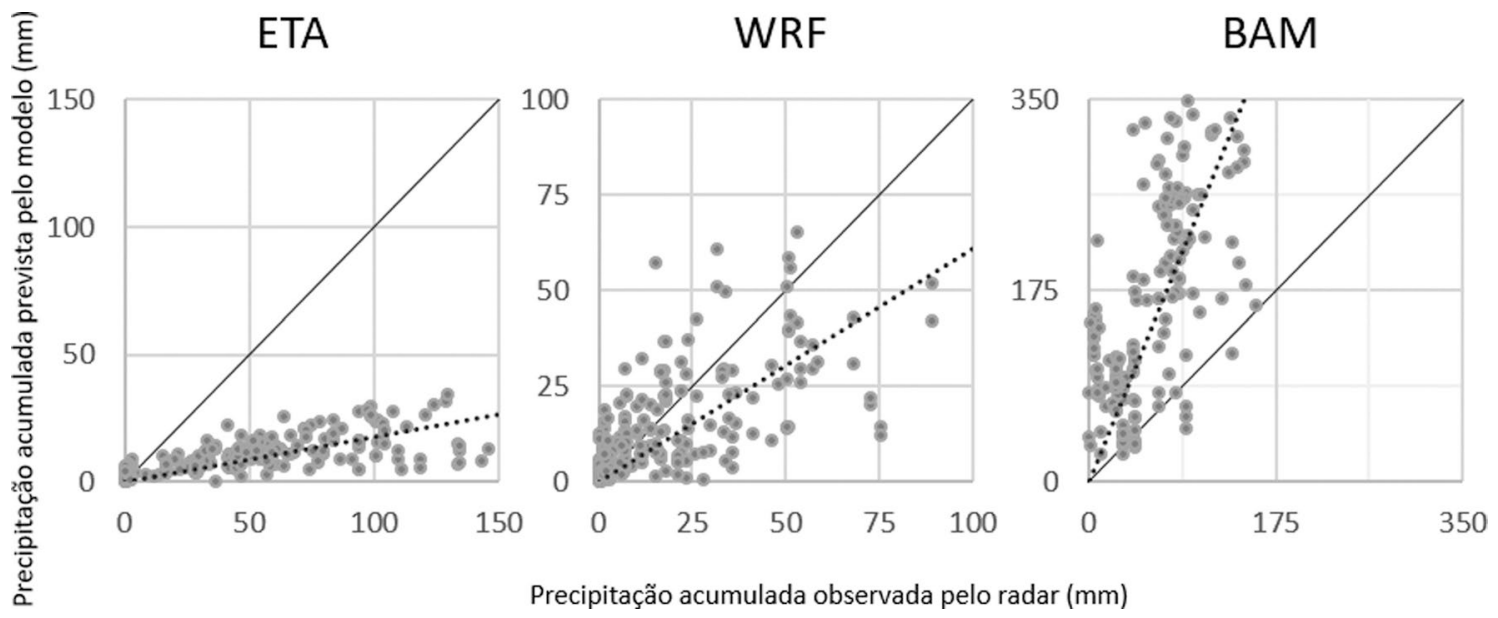

Figura 4 - Relação entre as precipitações acumuladas observadas pelo radar e previstas pelos modelos ETA (a), WRF (b) e BAM (c). 
Tabela 4 - Coeficiente angular da regressão linear para valores de precipitação acumulada.

\begin{tabular}{|c|c|c|c|c|c|c|}
\hline & \multicolumn{2}{|c|}{ ETA } & \multicolumn{2}{|c|}{ WRF } & \multicolumn{2}{|c|}{ BAM } \\
\hline & Radar & Pluviômetro & Radar & Pluviômetro & Radar & Pluviômetro \\
\hline Jaguari/Jacareí & 0,1770 & 0,2367 & 0,6105 & 0,5715 & 2,3296 & 2,4242 \\
\hline Cachoeira & 0,1716 & 0,2773 & 0,6658 & 0,7191 & 2,1257 & 2,1761 \\
\hline Atibainha & 0,1671 & 0,2395 & 0,6797 & 0,4941 & 1,9321 & 1,4285 \\
\hline Paiva Castro & 0,1906 & 0,2613 & 0,5746 & 0,5972 & 1,8443 & 2,0698 \\
\hline Posto Buenópolis & 0,1650 & 0,2700 & 0,6290 & 0,7921 & 1,6167 & 2,1037 \\
\hline Posto Valinhos & 0,1468 & 0,1940 & 0,5967 & 0,5358 & 1,3487 & 1,3206 \\
\hline Posto Atibaia & 0,1184 & 0,1412 & 0,6368 & 0,7414 & 1,7001 & 2,0960 \\
\hline
\end{tabular}

aumenta a incerteza sobre a proporção indicada pelo coeficiente angular da regressão linear. No caso dos modelos ETA e BAM, a magnitude do coeficiente angular era tão alta que mesmo considerando a dispersão dos dados, quase a totalidade dos valores apresentavam a mesma tendência de subestimar ou superestimar as observações. A análise do coeficiente angular é confirmada pelos resultados encontrados para a componente de viés do KGE (Fig. 6b) e pelo PBIAS Fig. 5a), que também indicam forte tendência do ETA de subestimação das observações, uma tendência leve do WRF no mesmo sentido e uma forte tendência do BAM de superestimar os eventos.
Os modelos apresentaram baixo desempenho para o KGE (Fig. 6d), sendo o modelo WRF o que obteve melhor resultado. Analisando suas componentes, é possível verificar que além do coeficiente de correlação, os modelos tiveram bom desempenho na parcela de variabilidade do índice. A componente de viés foi determinante para o mal desempenho pelo KGE, com valores muito baixos para o ETA e muito elevados para o BAM. Apesar do bom desempenho do WRF nessa componente, com valores próximos do ótimo, o modelo indicou valores significativos de erro pelo REQM (Fig. 5b), indicando uma possível ocorrência de erros compensatórios entre si. Esse

\section{PBIAS}

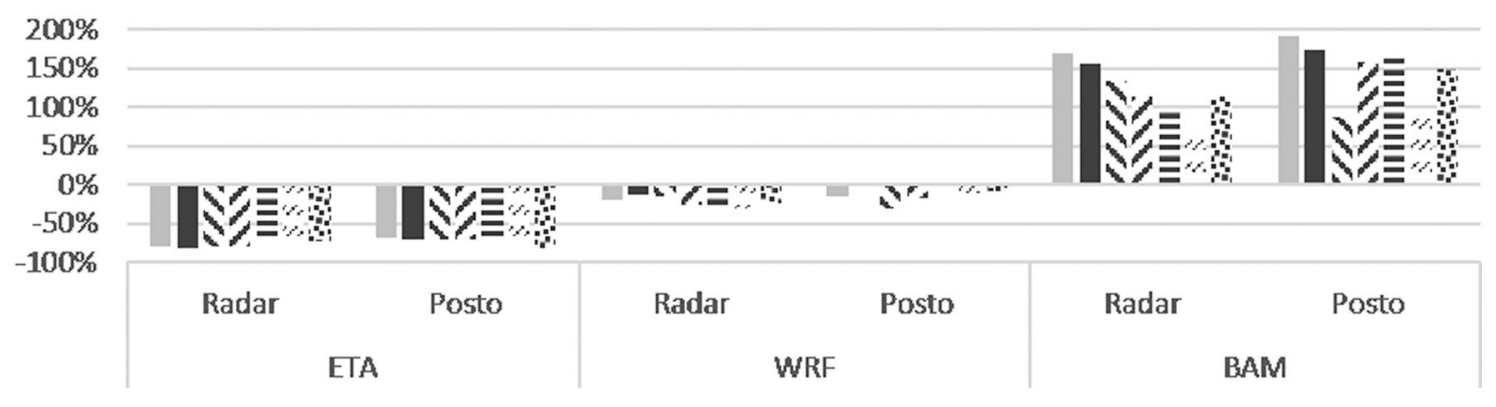

REQM

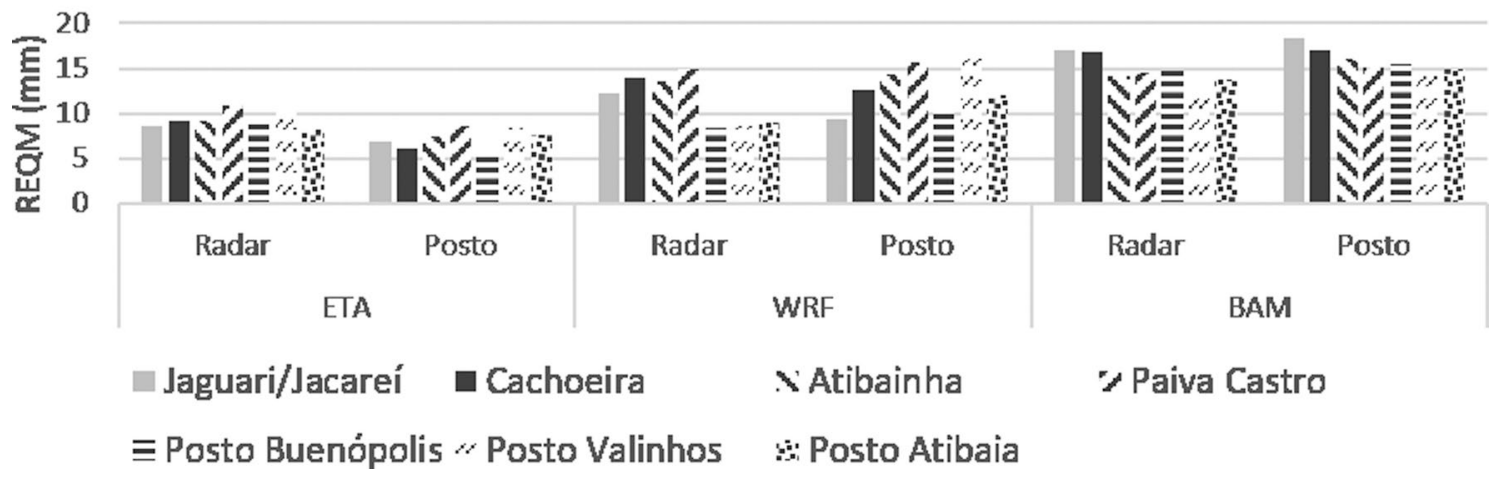

Figura 5 - Comparação dos valores de PBIAS (a) e REQM (b) para as séries completas de precipitação previstas. 
(a)
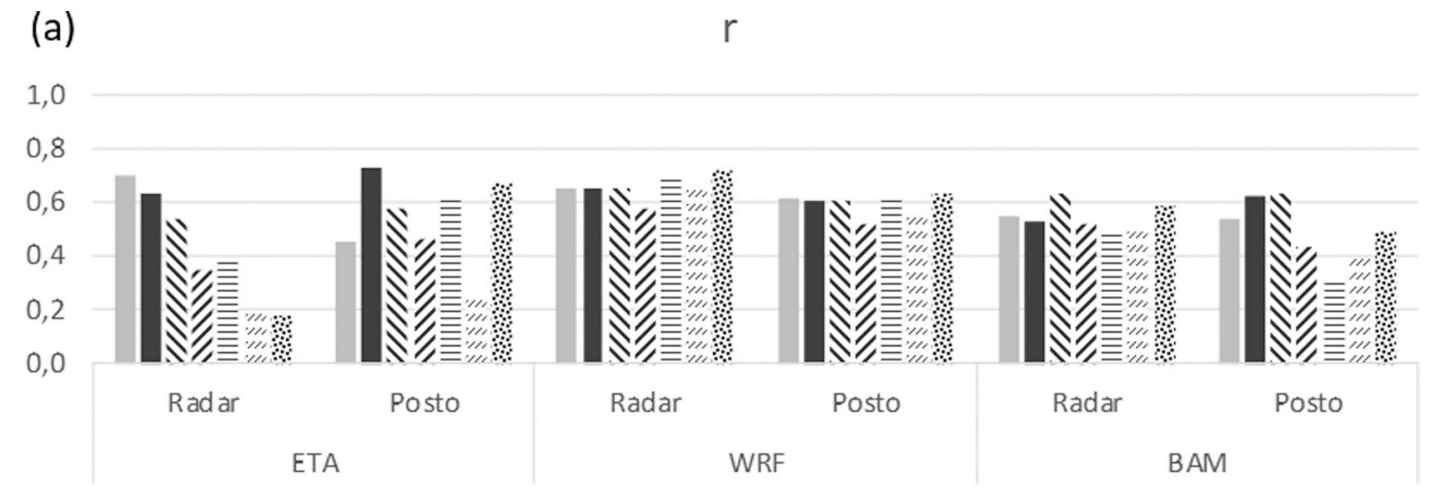

(b)

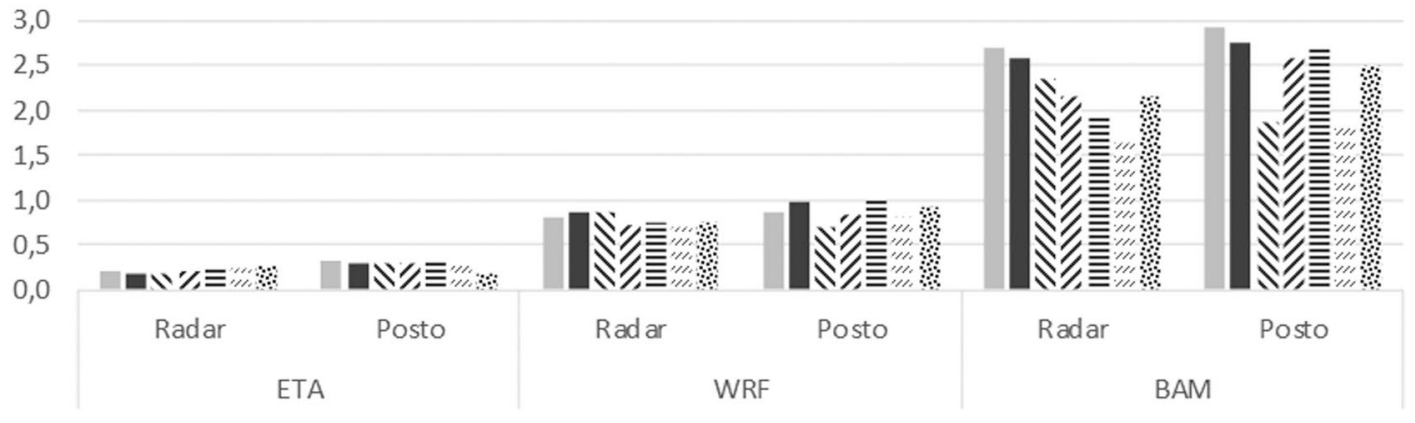

(c) $\gamma$

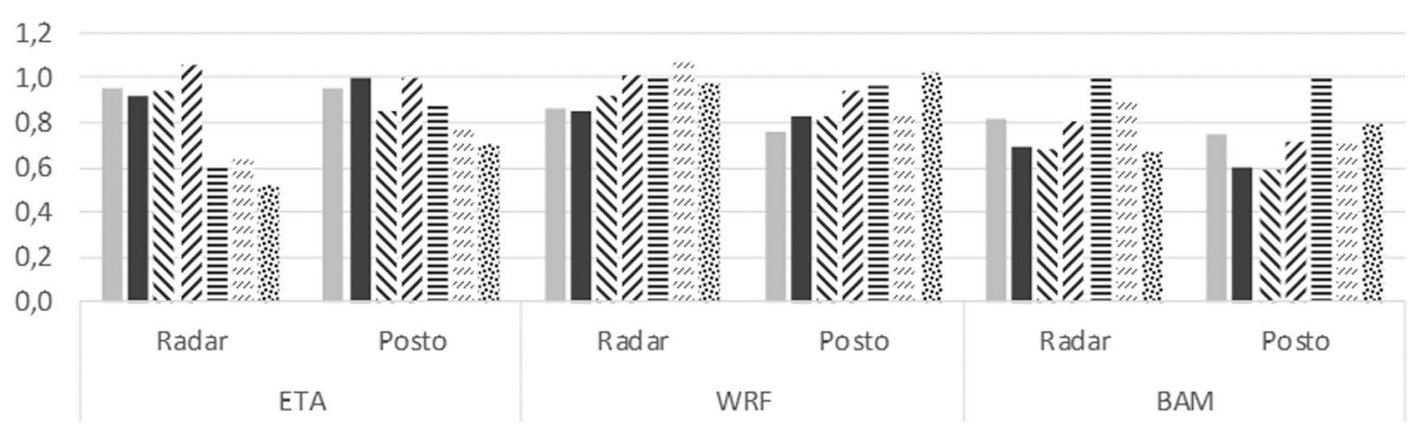

(d)

KGE

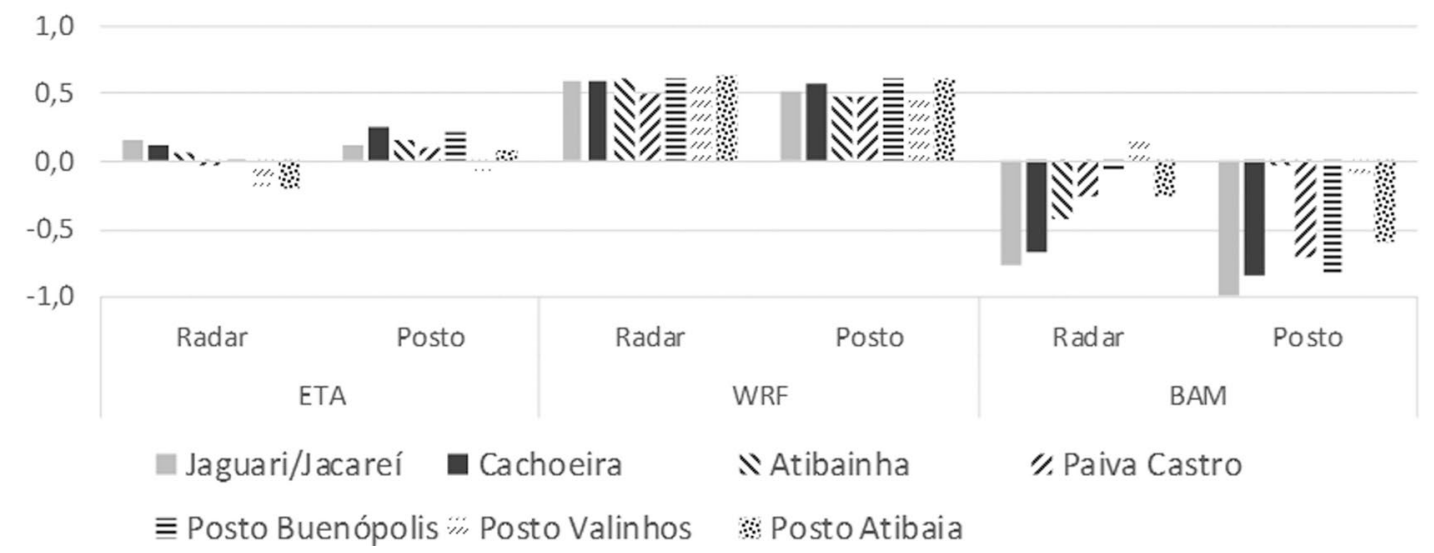

Figura 6 - Comparação das componentes linear (a), viés (b) e dispersão (c) do KGE (d) para as precipitações acumuladas dos três modelos. 
resultado é sugerido pela análise visual da dispersão dos valores previstos (Fig. 2b).

Os índices categóricos (Fig. 7) confirmam os resultados inferidos pelos demais índices e fornecem mais detalhes sobre o comportamento das previsões. O fBIAS confirma as tendências indicadas pelas análises de viés, indicando ainda que essa tendência ocorre não apenas na magnitude, mas também na frequência com que os modelos preveem eventos. O modelo BAM tende a indicar a ocorrência de eventos com uma frequência maior com a que realmente ocorrem, fato também confirmado pelos valores elevados do FAR para esse modelo. Os modelos ETA e WRF apresentaram valores elevados para POD, com baixo FAR, para pequenos limiares de precipitação.
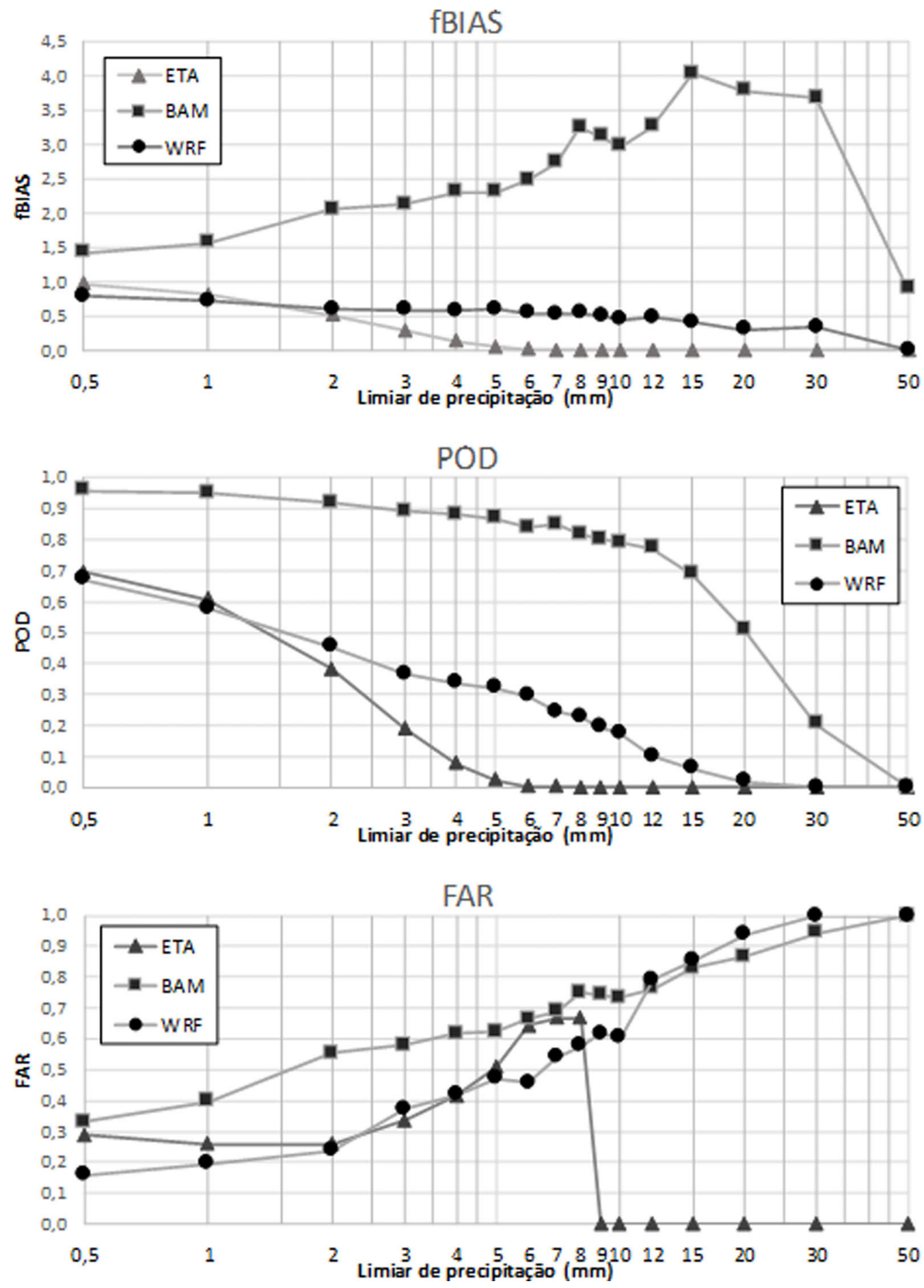

Figura 7 - Comparação dos índices categóricos para as séries completas dos três modelos. 
Com o aumento do limiar, o POD decresce rapidamente, sendo o modelo ETA praticamente incapaz de detectar eventos acima de $5 \mathrm{~mm}$, enquanto o modelo WRF chega próximo da nulidade com cerca de $20 \mathrm{~mm}$ de limiar. Para limiares muito elevados, no entanto, os índices categóricos não foram conclusivos, a maioria dos eventos é categorizada como ausência de chuva e o denominador tende a 0 , distorcendo os resultados ou impossibilitando seu cálculo.

As análises realizadas acima mostram uma diferença entre os valores para as comparações com observações de radar e de pluviômetro. Alguma diferença era esperada, dada a natureza de cada técnica, mas em alguns casos se apresentou bastante significativa. Essas diferenças coincidem com os valores de menor correlação entre as previsões e observações, como é o caso da bacia correspondente ao Posto Valinhos, no período utilizado nas análises do modelo ETA. Como já mencionado, o coeficiente de correlação de Pearson sofre grande influência do horizonte de análise. Esse estudo dispôs de alguns meses de previsão para cada um dos modelos. Ao se comparar períodos mais longos, a correlação entre as observações de radar e de pluviômetros é forte. Nesse caso, o coeficiente de correlação entre as variáveis é de 0,8067 , com leve tendência do radar a subestimar os valores do pluviômetro $(a=0,8343)$.

\section{Conclusões}

O estudo avaliou, através de diversos índices, as previsões dos modelos ETA (resolução horizontal $40 \mathrm{~km}$ ), WRF (resolução horizontal 5 km) e BAM (resolução horizontal $20 \mathrm{~km}$ ). As análises indicaram que existe maior correlação com as observações nos primeiros dias de previsão, com significativo decrescimento ao longo do horizonte de previsão. A correlação foi mais forte ao se avaliar as precipitações acumuladas para todo o período de previsão, sendo maior nas comparações com o radar que nas comparações com o pluviômetro.

A avaliação do coeficiente angular da regressão linear, do PBIAS e da componente de viés do KGE indicou forte tendência de subestimar as observações por parte do modelo ETA e forte tendência de superestimar as observações por parte do modelo BAM. O modelo WRF indicou uma tendência leve de subestimar os valores, sendo necessária cautela na interpretação dessa tendência, visto que a correlação é apenas moderada. As tendências verificadas em termos de magnitude, também foram confirmadas em termos de frequência, através do fBIAS.

Os índices categóricos se mostraram úteis na análise, revelando informações adicionais sobre as previsões. $\mathrm{O}$ POD e o FAR indicaram um bom desempenho dos modelos ETA e WRF para baixas precipitações, perdendo desempenho na ocorrência de eventos mais intensos.

A análise proposta mostrou-se sensível aos valores extremos e horizonte das previsões, sugerindo que con- clusões mais robustas possam ser obtidas dispondo de um número maior de previsões.

Os modelos de previsão se apresentam como uma ferramenta útil na gestão de recursos hídricos, mas o uso de previsões para a tomada de decisão deve ser acompanhado de avaliação das incertezas e riscos envolvidos em cada caso. O risco ao se superestimar os valores é frequentemente diferente do risco ao subestimá-los, sendo essa informação um importante elemento na escolha do modelo a ser utilizado. O uso conjunto de diferentes índices de avaliação permite uma visão mais completa do comportamento dessas previsões.

\section{Agradecimentos}

Os autores agradecem à Sabesp, pela disponibilização dos dados de sua rede telemétrica. Ao Msc. Cristiano de Pádua Milagres Oliveira, pelo apoio na obtenção das previsões, à Msc. Carla Voltarelli Franco da Silva, pelo apoio no tratamento das informações geoespaciais e à toda a equipe do LabSid - USP. Aos revisores, pelas suas importantes contribuições ao trabalho.

\section{Referências}

ANA - Agência Nacional de Águas; DAEE - Departamento de Águas e Energia Elétrica. Resolução conjunta ANA/DAEE n. 925, de 29 de maio de 2017. 2017a. Disponível em: http://arquivos.ana.gov.br/resolucoes/2017/925-2017.pdf. Acesso em 29 de Janeiro de 2018.

ANA - Agência Nacional de Águas; DAEE - Departamento de Águas e Energia Elétrica. Resolução conjunta ANA/DAEE n. 926, de 29 de maio de 2017. 2017b. Disponível em: http://arquivos.ana.gov.br/resolucoes/2017/926-2017.pdf. Acesso em 29 de Janeiro de 2018.

BAEZ-VILLANUEVA, O.M; BIGIARINI, M.Z.; RIBBE, L.; NAUDITT, A.; GIRALDO-OSORIO, J.D.; THINH, N.X. Temporal and spatial evaluation of satellite rainfall estimates over different regions in Latin-America. Atmospheric Research, v. 213, p. 34-50, 2018.

BARROS, M.T.; LOPES, J.E.; ZAMBON, R.C.; FRANCATO, A.L.; BARBOSA, P.S.; ZANFELICE, F.R. Climate flow forecast model for the Brazilian hydropower system. World Environmental and Water Resources Congress 2009: Great Rivers, p. 1-9, 2009.

BLACK, T.L. The new NMC mesoscale Eta model: Description and forecast examples. Weather and forecasting, v. 9, n. 2, p. 265-278, 1994.

CARDOSO, B.C.T. Estudo da Eficiência da Previsão Numérica do Tempo de Curto Prazo Para o Município de Maceió/AL, Utilizando o Modelo WRF. Dissertação. Universidade Federal de Alagoas, 2018.

CATALDI, M.; MACHADO, C.O.; GUILHON, L.G.F.; CHOU, S.C.; GOMES, J.L.; BUSTAMANTE, J.F. Análise das previsões de precipitação obtidas com a utilização do modelo Eta como insumo para modelos de previsão semanal de 
vazão natural. Revista Brasileira de Recursos Hídricos, v. 12 , n. 3, p. 5-12, 2007.

CHOU, S.C. Modelo regional ETA. Climanálise-Boletim de Monitoramento e Análise Climática, v. 1, n. especial, 1996. Disponível em: http://climanalise.cptec.inpe.br/ rcli $\mathrm{manl} /$ boletim/cliesp10a/27.html. Acesso em 29 de Maio de 2019.

ELLIOTT, A.C.; WOODWARD, W.A. Statistical Analysis Quick Reference Guidebook: With SPSS examples. Sage, Thousand Oaks, 2007.

FIGUEROA, S.N.; BONATTI, J.P.; KUBOTA, P.Y.; GRELL, G.A.; MORRISON, H.; BARROS, S.R.; et al. The Brazilian global atmospheric model (BAM): performance for tropical rainfall forecasting and sensitivity to convective scheme and horizontal resolution. Weather and Forecasting, v. 31, n. 5, p. 1547-1572, 2016.

GUPTA, H.V.; KLING, H.; YILMAZ, K.K.; MARTINEZ, G.F. Decomposition of the mean squared error and NSE performance criteria: Implications for improving hydrological modelling. Journal of hydrology, v. 377, n. 1-2, p. 80-91, 2009.

HABIB, E.; KRAJEWSKI, W.F.; CIACH, G.J. Estimation of rainfall interstation correlation. Journal of Hydrometeorology, v. 2, n. 6, p. 621-629, 2001.

INSTITUTO NACIONAL DE PESQUISAS ESPACIAIS. CPTEC lança novo modelo global e investe para se manter como referência mundial em previsões para a região tropical. Informativo INPE, n. 6, 2016.

KESSLER, Edwin; NEAS, Barbara. On correlation, with applications to the radar and raingage measurement of rainfall. Atmospheric Research, v. 34, n. 1-4, p. 217-229, 1994.

KLING, H.; FUCHS, M.; PAULIN, M. Runoff conditions in the upper Danube basin under an ensemble of climate change scenarios. Journal of Hydrology, v. 424, p. 264-277, 2012.

LORIATO, A.G.; SALVADOR, N.; LORIATO, A.A.B.; SOKOLOV, A.; NASCIMENTO, A.P.; et al. Inventário de emissões com alta resolução para a região da grande Vitória utilizando o Sistema de Modelagem Integrada WRFSMOKE-CMAQ. Revista Brasileira de Meteorologia, v. 33, n. 3, p. 521-536, 2018.

MESINGER, F.; JANJIĆ, Z.I.; NIČKOVIĆ, S.; GAVRILOV, D.; DEAVEN, D.G. The step-mountain coordinate: Model description and performance for cases of Alpine lee cyclogenesis and for a case of an Appalachian redevelopment. Monthly Weather Review, v. 116, n. 7, p. 1493-1518, 1988.

MESINGER, F.; CHOU, S.C.; GOMES, J.L.; JOVIC, D.; BASTOS, P.; BUSTAMANTE, J.F.; et al. An upgraded version of the Eta model. Meteorology and Atmospheric Physics, v. 116, n. 3-4, p. 63-79, 2012.

MOURA, R.G.D.; HERDIES, D.L.; MENDES, D.; MENDES, M.C.D. Avaliação do modelo regional ETA utilizando as análises do CPTEC e NCEP. Revista Brasileira de Meteorologia, v. 25, n. 1, p. 46-53, 2010.
MUKAKA, M.M. A guide to appropriate use of correlation coefficient in medical research. Malawi Medical Journal, v. 24, n. 3, p. 69-71, 2012.

MURPHY, A.H. What is a good forecast? An essay on the nature of goodness in weather forecasting. Weather and forecasting, v. 8, n. 2, p. 281-293, 1993.

PELLEGRINI, C.C.; ARAUJO, C.; REIS, A.; PIMENTA, F.M.; ASSIREU, A.T. Análise do desempenho do modelo WRF num episódio de vento intenso e persistente num grande reservatório tropical. Revista Brasileira de Meteorologia, v. 34, n. 1 p. 121-138, 2019.

PEREIRA FILHO, A.J.; CRAWFORD, K.C.; HARTZELL, C.L. Improving WSR-88D hourly rainfall estimates. Weather and Forecasting, v. 13, n. 4, p. 1016-1028, 1998.

ROCHA FILHO, K.L. Modelagem Hidrológica da Bacia do Rio Pirajuçara com TOPMODEL, Telemetria e Radar Meteorológico. Tese de Doutorado, Universidade de São Paulo, 2010.

SABESP Companhia de Saneamento Básico do Estado de São Paulo. Estudos de Cheias para Avaliação dos Volumes de Espera dos Aproveitamentos do Sistema Cantareira. Sabesp, São Paulo, 2009.

SCHARDONG, A.; MÉLLO JÚNIOR, A.V.; ROBERTO, A.N.; PORTO, R.L.L. Desempenho do modelo AcquaNetXL na alocação de água em sistemas de recursos hídricos complexos. Engenharia Agrícola, v. 29, n. 4, p. 705-715, 2009.

SCHARDONG, A.; SIMONOVIC, S.P.; GARCIA, J.I.B. O possível efeito de mudanças climáticas e suas incertezas sobre afluências em sistemas de recursos hídricos. REGA, v. 11, n. 2, p. 53-65, 2014.

SAISP Sistema de Alerta a Inundações de São Paulo. Chuva Observada na Área do Radar - CAPPI. Disponível em https://www.saisp.br/estaticos/sitenovo/produtos.xmlt\#a2. Acesso em: 20 de Maio de 2019.

SALIO, P.; HOBOUCHIAN, M.P.; SKABAR, Y.G.; VILA, D. Evaluation of high-resolution satellite precipitation estimates over southern South America using a dense rain gauge network. Atmospheric Research, v. 163, p. 146$161,2015$.

SKAMAROCK, W.C.; KLEMP, J.B.; DUDHIA, J.; GILL, D.O.; BARKER, D.M.; WANG, W.; POWERS, J.G. A Description of the Advanced Research WRF Version 3, NCAR Technical Note. National Center for Atmospheric Research, Boulder, 2008.

TAYLOR, R. Interpretation of the correlation coefficient: a basic review. Journal of Diagnostic Medical Sonography, v. 6, n. 1, p. 35-39, 1990.

License information: This is an open-access article distributed under the terms of the Creative Commons Attribution License (type CC-BY), which permits unrestricted use, distribution and reproduction in any medium, provided the original article is properly cited. 